\title{
Luminosity Function of the Perigalactocentric Region
}

\author{
Vijay K. Narayanan \\ Andrew Gould 1 \\ D. L. DePoy \\ Dept. of Astronomy, The Ohio State University, Columbus, OH 43210 \\ vijay@payne.mps.ohio-state.edu \\ gould@payne.mps.ohio-state.edu \\ depoy@payne.mps.ohio-state.edu
}

\begin{abstract}
We present $H$ and $K$ photometry of $\sim 42,000$ stars in an area of $\sim 250 \operatorname{arcmin}^{2}$ centered on the Galactic center. We use the photometry to construct a dereddened $K$ band luminosity function (LF) for this region, excluding the excessively crowded inner $2^{\prime}$ of the Galaxy. This LF is intermediate between the LF of Baade's window and the LF of inner $2^{\prime}$ of the Galactic center. We speculate that the bright stars in this region have an age which is intermediate between the starburst population in the Galactic center and the old bulge population. We present the coordinates and mags for 16 stars with $K_{0} \leq 5$ for spectroscopic follow up.
\end{abstract}

Subject Headings: Galaxy: center-infrared: stars-stars: luminosity function - ISM: dust, extinction

submitted to The Astrophysical Journal: March 12, 1996

Preprint: OSU-TA-4/96

\footnotetext{
${ }^{1}$ Alfred P. Sloan Foundation Fellow
} 


\section{Introduction}

The $2.2 \mu \mathrm{m}$ luminosity functions (LFs) of Baade's window (BW) and the inner $2^{\prime}$ of the Galactic center (GC) are markedly different at the bright end. The GC LF has a substantially higher fraction of stars brighter than $K_{0}=5.5$, but it is nearly identical to the BW LF at the faint end for $K_{0}>7.0$. This suggests that the GC contains a population of younger stars in addition to an older population of red giants similar to the one in BW (Haller 1992; Blum et al. 1996). Here, we present $H$ and $K$ photometry of $\sim 42,000$ stars in an area $\sim 16^{\prime} \times 16^{\prime}$ centered on the GC. In our analysis, we exclude the inner $2^{\prime}$ and refer to the remaining area as the Perigalactocentric region (PGC). We construct the $K$ band LF of the PGC by individually dereddening each star using its observed $(H-K)$ colors. We find that the PGC does not contain any super bright stars of the type found in the GC. For $K_{0}<6.0$, the GC LF has a significant excess over the PGC LF. In comparing the PGC with BW, we find that for BW the contamination by the foreground distribution of $M$ giants is significant for $K_{0}<7.0$, while for the PGC it is negligible. Even if the contamination in BW is neglected, there is a still a clear excess of stars in the PGC over BW in the range $4.0<K_{0}<7.0$. We conclude that the luminosity function of the PGC is intermediate between that of the GC and Baade's window.

\section{Observations and Mosaic construction}

We constructed mosaic images of the GC and PGC in $H$ and $K$ bands by stitching together $8 \times 19=152$ overlapping images. The grid of images was taken on UT 1995 June 10 and 11 using the Ohio State Infrared Imaging Spectrograph (OSIRIS; DePoy et al. 1993) at the $1.8 \mathrm{~m}$ Perkins Telescope, located on Anderson Mesa near Flagstaff, Arizona. OSIRIS uses a $256 \times 256$ NICMOS III array. All the images were taken under photometric conditions. The plate scale is $\sim 00^{\prime \prime} 63$ per pixel $\left(\sim 155^{\prime \prime}\right.$ field of view). Images in the same row are offset by $\sim 50^{\prime \prime}$ while those in the same column are offset by $\sim 120^{\prime \prime}$. The images were exposed successively without any intervening sky exposures. The seeing was between 1."4 and 2." 4 in $H$ and between 2." 1 and 2."8 in $K$. We constructed the $H$ and $K$ mosaics independently. A standard star P565-C was observed both before and after the observations in the $H$ band and after the observations in the $K$ band. Each exposure was $\sim 1.1$ seconds long. We coadded 10 individual exposures to form each individual image. The sky image formed from 
the median of the standard star images was subtracted from each of the raw images. Finally, we divided the images by a flatfield formed from the median of the images of a white screen mounted to the telescope.

We aligned the images geometrically and photometrically, before making the final mosaic. We cross-correlated every pair of overlapping images in order to determine 4 parameters that relate the images: a vertical offset, a horizontal offset, a sky offset, and the relative intensity normalization (due to differences in atmospheric transparency and exposure times). We obtained a global solution for these parameters by weighting each pair according to the number of overlapping pixels. Bad pixels and saturated stars were rejected before the cross correlation. The overlapping sections of the images were shifted by fractions of a pixel using linear interpolation.

The global solution for the geometric and photometric offsets between the images was used to construct the mosaic in the $H$ and $K$ bands. In combining the pixels, we averaged all the contributing pixel values, after rejecting the pixel values that were more than $5 \sigma$ away from the mean of the remaining pixel values, where $\sigma$ is computed assuming photon counting statistics. In the case of bright stars, we accepted all the contributing pixel values to ensure that the flux of the star is conserved. This procedure does not eliminate cosmic ray events when they lie on top of bright stars. However the short exposure times of the images should ensure that the cosmic ray events do not significantly contaminate the image. We verified this by visually scanning the image. Some of the individual $H$ band images contain interference fringes from variable $\mathrm{OH}$ emission with typical amplitude of $\sim 6 \mathrm{ADU}$ and width of $\sim 15$ pixels. Fringes of this type are often removed from $H$ band images by chopping to the sky. As noted above, we did not chop. However, the typical amplitude of the fringes in the mosaic image is only $\sim 2$ ADU (since typically, each pixel in the final mosaic is an average of pixels in 3 individual images), corresponding to $\mathrm{H} \sim 19$ mag $\operatorname{arcsec}^{-2}$ and hence too small to affect the results.

The $H$ and $K$ mosaics are shown in Figures 1 and 2 respectively. The $K$ mosaic is offset by $\sim 40^{\prime \prime}$ to the west relative to the $H$ mosaic. Excluding the rim of the mosaic where there is information from only one image, about $60 \%$ area of each mosaic is constructed from 3 overlapping images and the other $40 \%$ from 6 images. The typical sky level is $\sim 14 \mathrm{mag} \operatorname{arcsec}^{-2}$ in the $H$ mosaic and $\sim 12 \mathrm{mag} \operatorname{arcsec}^{2}$ in the $K$ mosaic. One ADU corresponds to $\sim 21 \mathrm{mag} \operatorname{arcsec}^{-2}$ in $H$ and $\sim 20.4 \mathrm{mag} \operatorname{arcsec}^{-2}$ in $K$. There is a difference of $\sim 2$ to 3 ADU across the seams in the final mosaic, of order $1 \%$ of the sky. 


\section{Photometry}

We initially identified $\sim 28,000$ candidate stars in the $H$ mosaic using the DAOFIND routine of DAOPHOT (Stetson 1987). The extinction pattern in the images has many patchy and filamentary structures on small scales. For stars in these highly obscured patches, the local sky determined from an annular region around the star by DAOPHOT was often greater than the peak flux of the star itself. These stars were either not identified or photometered incorrectly by DAOPHOT. We therefore manually identified the stars not found by DAOFIND by displaying the $H$ mosaic in various stretches of brightness levels. We used the $K$ mosaic to help identify the faint stars in some heavily obscured regions. However, the sample of stars is essentially $H$ band selected, because of better seeing in $H$. Finally, we identified a total of $\sim 65,000$ candidate stars.

The photometry was carried out by extracting small, overlapping sections of $100 \times$ 100 pixels from the $H$ and $K$ band mosaics. The overlap between the neighboring sections is 5 pixels in both the horizontal and vertical directions. There are 342 such sections in each band. Each of these sections was photometered independently. In each section, only the stars that lie within the inner $95 \times 95$ pixels were accepted in the final photometry list. We measured the $H$ and $K$ magnitudes of the stars by fitting the stellar profiles to an appropriate point spread function (PSF) and the local sky. The instrumental magnitudes determined from this PSF fitting were converted to apparent magnitudes using aperture photometry of PSF stars compared to the standard stars.

The observations were carried out at $\sim 2$ air masses, while the standard star was observed at $\sim 1$ air mass. We therefore applied an air mass correction of 0.1 mag in both $H$ and $K$ bands. There is a residual uncertainty of $\sim 0.03$ mag in the photometric zero points because of the uncertainty in the appropriate value of this correction.

\section{$3.1 \quad H$ band}

The seeing in $H$ band changed significantly during the approximately 2 hour period of observations. Therefore, we identified 98 stars that were well isolated and bright enough that they were not contaminated by the light from neighboring stars. These 
PSF stars are spread approximately uniformly throughout the $H$ image. The stars in each of the 342 small sections were photometered using a PSF that was most similar to the PSF for that section. Except in a few cases where the seeing changed abruptly, the PSF nearest to a section was adequate for this purpose.

We fit all the stars in a section simultaneously. Starting from the approximate coordinate locations, we first fit each star to 4 parameters - 3 parameters that model the sky (including a constant offset and linear terms in the horizontal and vertical directions) and 1 parameter for the PSF normalization. With these parameters, we then find the best values for the coordinate locations of the star by shifting the stellar profile by fractional pixel amounts and minimizing the $\chi^{2}$ difference between the fit and the image. This best fit for the star is then subtracted from the image. All the stars are fitted and subtracted from the original image in this manner to create a residual image.

The brightest star was then added to this residual image. It was again fit for all the 6 parameters that define the PSF normalization, the local sky, and the best coordinate locations in the same manner as described above, and this best fit was again subtracted from the residual image. This procedure was repeated for all the other stars in order of their decreasing brightness as determined from the first pass. Therefore, before we fit for any star, all its brighter neighbors would have already been measured and their fluxes subtracted, thereby reducing the contamination from neighbors.

\section{$3.2 K$ band}

The $K$ mosaic is distorted by $\sim 0.1 \%$ with respect to the $H$ mosaic. We determined a linear transformation between the $H$ and $K$ mosaics, using the locations of 142 bright stars. The distortion was largely removed by this transformation, as was verified by the residuals of the fit. The seeing in $K$ band is worse than the $H$ band. There are only a few isolated stars that are not contaminated by neighboring stars. However, the seeing remained approximately constant throughout the $K$ band observations. Therefore, all the stars in the $K$ band were fit to 5 different PSFs in a manner similar to the $H$ photometry. In this fitting procedure however, we assumed that the transformed locations of the stars are accurate enough. For each star photometered in $H$, we assumed that the $K$ mosaic also contained the star at the corresponding 
transformed location. Therefore, each star was fitted for 4 parameters only. We take the average of these 5 measurements (after rejecting $3 \sigma$ outliers) to be the $K$ mag. We estimate the error in the $K$ photometry as the standard deviation of the 5 measurements averaged over the stars in a given mag bin (Fig. 3).

\subsection{Errors in the Photometry}

$H$ and $K$ mag are measured for $\sim 42,300$ stars in the entire field. The other candidates are either misidentifications or are too faint in one of the bands to be measured by this method. The errors in $H$ band are assumed to be the same as the errors in $K$ band.

The photometric errors in both bands depend on the crowding of stars. Very close to the GC, the photometry is limited by our ability to identify separate stars. Excessive crowding of stars, together with the relatively poor seeing made it difficult to separate the objects into individual stars. The results reported here are based on photometry of regions more than $2^{\prime}$ away from the GC.

\section{Dereddened magnitudes}

The observed $(H-K)$ color of a PGC star is the sum of the intrinsic color $(H-K)_{0}$ and the selective extinction coefficient, $E(H-K)$. Therefore, we deredden the PGC stars individually using the observed $(H-K)$ colors and an appropriate color-mag relation. We use the near infrared extinction law determined by Mathis (1990) to compute the total extinction coefficients $A_{K}$ and $A_{H}$. Specifically, we assume $A_{H} / A_{K}=1.66$ and $A_{K}=1.5 E(H-K)$.

We assume that the intrinsic color-mag relation for stars in PGC (corrected for

extinction) is the same as that of stars in BW. We fit a color-mag relation for stars in BW using the photometry provided to us by J. Frogel (1995, private communication) to obtain the following analytic relation,

$$
\begin{gathered}
K_{0}=-9(H-K)_{0}+10.7 \quad\left(K_{0}<8.5\right), \\
K_{0}=-50(H-K)_{0}+18.0 \quad\left(K_{0}>8.5\right),
\end{gathered}
$$


All stars with $E(H-K)<0.6$ are assumed to be foreground stars. There are 10 stars that are saturated either in the $H$ or $K$ mosaic, or both. Therefore, we divided the $H$ mosaic by the $K$ mosaic and determined the colors of these stars by estimating the color at the wings, where the pixel counts are not saturated. We estimated the colors at the wings by comparing them with nearby stars that were not saturated. Only one of these 10 stars is sufficiently reddened to be a PGC star.

\section{Luminosity function of the PGC}

The PGC suffers from very patchy extinction. As a result, the completeness level of our photometry can change drastically throughout the imaged region. Therefore, we identified 6 regions around the GC that have moderate $\left(A_{K} \sim 2.7\right)$ and roughly homogeneous extinction (collectively called Reg1). While Reg1 does contain a few patches of high extinction, these patches are very small and do not affect our results. Reg1 encloses about $5 \%$ of the total area of the PGC. The 6 regions comprising Reg1 are chosen at more than $2^{\prime}$ away from the GC, so the photometry does not suffer from the severe crowding of the GC. All the Reg1 stars with $K_{0} \leq 8.0$ were examined individually and the bad photometry cases were rejected. The color-mag diagram of patch 1 of Reg1 is shown in Figure 4. In order to be able to compare the Reg1 LF with that of BW and GC, we normalize the star counts in a given region by the integrated $K$ band flux (corrected for extinction) falling in that region. We determine the dereddened flux as follows. In each of the 6 regions comprising Reg1, we compute the mean $\bar{E}(H-K)$ of stars from the mean offset in $(H-K)$ relative to the giants in BW. We then find the total flux within the region and multiply this by $10^{0.4 \bar{A}_{K}}$, where $\bar{A}_{K}=1.5 \bar{E}(H-K)$. We estimate the true sky by measuring the counts in heavily extincted regions. This gives an upper limit to the true sky as there is some $K$ light from the bulge even in heavily extincted regions. This sky is then used to estimate the counts due to bulge light in lightly extincted regions with a small fractional error due to the uncertain sky. By comparing the extinctions in these 2 regions (measured from the reddening of bright stars), we can accurately estimate the small amount of $K$ light from the bulge in the heavily extincted region. We estimate the errors in this procedure to be $\sim 3$ ADU per pixel. This leads to an uncertainty of $\sim 2 \%$ in the total flux, negligible compared to other errors. 
To verify the accuracy of this LF of the PGC, we also identified another 22 regions of moderate reddening (Reg2), covering another $20 \%$ of the total area of the PGC. All stars with $K_{0} \leq 6.5$ in these regions were examined individually and the LF was constructed in a similar manner. The LFs determined for Reg1 and Reg2 are shown in Table 1 and are plotted in Figure 5. The two LFs are identical within Poisson errors.

The $H$ and $K$ mosaics give the visual impression of a disk-like feature approximately aligned with the Galactic plane (Figs. 1 and 2). To test for the reality of this feature, we selected all the Reg1 regions from within the apparent disk and all the Reg2 regions from outside it. The centroids and areas of the 6 patches comprising Reg1 and the 22 patches comprising Reg2 are in Table 2 and Table 3 respectively. The locations and geometries of these patches are shown in Figure 6. Although a few patches lie close to each other, they are reddened by different amounts. The LFs of these two regions are the same, indicating that the stellar populations at least of these two regions are indistinguishable.

\section{Completeness limit of the LF}

The LF is based on stars selected in the $H$ mosaic. Stars were identified first by using the DAOPHOT star finding routine DAOFIND and then by scanning the images manually to find the stars missed by DAOFIND. Based on the tests described below, we conclude that the LF is complete up to $K_{0}=8$.

We added 10 artificial stars per half mag bin in $H$ at random locations in each patch. We ran DAOFIND on the resulting images to determine the fraction of stars detected. For each undetected star, we judged whether we would have found the star in the manual search. Figure 7 shows the completeness of the DAOFIND and combined (DAOFIND + manual) searches. The combined search is complete for $H<12.5$ and $\sim 80 \%$ complete at $H=14$.

The DAOFIND completeness limit is objective, but the DAOFIND + manual search limit depends in part on subjective judgement. To test whether this procedure reproduces the actual manual detection rate, we compared the fraction of stars that were undetected by DAOFIND but were found manually in the real data, with the

same fraction in the artificial-star sample. The results of this test are shown in Figure 
8. The sample star list fractions lie within $1 \sigma$ of the artificial star list fractions for all $H$. This consistency check confirms the accuracy of the combined (DAOFIND + manual) detection efficiency.

To construct the LF, we weighted each detected star by the inverse of the completeness fraction corresponding to its $H$ mag. The patches typically have $A_{H} \sim 4.5$. Therefore, the LF is fully complete for $K_{0}<8$. Due to the weighting procedure, we also expect the LF to be statistically complete for $8<K_{0}<9$.

Some of the stars in the sample star list are bad identifications. For $K_{0}<8$, we found these by checking each star individually. For $K_{0}>8$, we applied the following statistical corrections. We selected 100 stars randomly in each of the mag ranges $8<K_{0}<9,9<K_{0}<10$ and $10<K_{0}<11$. These stars were examined individually and bad identifications were rejected. In these 3 mag ranges, we found $15 \%, 22 \%$ and $22 \%$ of the total sample of stars to be bad identifications. For $K_{0}>11$, we assumed the bad identifications to be $22 \%$. We therefore multiplied the LF in these mag ranges by the fraction of good identifications.

\section{Comparison to BW and GC LFs}

We first compare the normalized $K$ band LF of the PGC with that of BW (Fig. $9)$. For $K_{0}<6.5$, we use the information from both Reg1 and Reg2 to improve the statistics. The LF for $K_{0}>6.5$ is constructed from Reg1 only. We use the BW LF from Tiede et al. (1995), which is complete in the range $5.5 \leq K_{0} \leq 16$. The bright end $\left(5.5 \leq K_{0} \leq 9.0\right)$ is derived by Frogel \& Whitford (1987) (hereafter referred to as FW87), from photometry of an unbiased subsample of the complete $M$ giant surveys by Blanco et al. (1984) and Blanco (1986). These surveys also included 10 Long period variables (LPVs). For $9.0 \leq K_{0} \leq 12.5$, the BW LF is based on the data of DePoy et al. (1993), who conclude that the $M$ giants selected from the grism surveys by FW87 include essentially all stars with $K_{0} \leq 10.0$, and that the FW87 field toward BW does not contain any other luminous stars. To compare the PGC and BW LFs, we normalize the latter to the total flux as follows. First, we compute the total flux of the stars in the LF which have $M_{\text {bol }}<-1.2$ (corresponding to $K_{0}<11.0$ at a distance to the GC of $R_{0}=8.0 \mathrm{kpc}$ ). FW87 and Frogel (1988) conclude that this flux accounts for $60 \%$ of the total $K$ band light. We therefore divide this flux by 0.6 to obtain the total flux, and use it to normalize the BW LF. Figure 9 also shows the 
GC LF obtained by Blum et al. (1996), who determined the relative normalization of the BW LF and GC LF.

Table 1 gives the 3 LFs together with the actual number of stars in each half mag bin on which the LFs are based. Unlike the GC, the PGC LF does not extend brighter than $K_{0}=4.0$. In fact, we did not find a single star with a dereddened mag $K_{0}<4.0$ in either Reg1 or Reg2. In the entire field of the PGC, there was only one star in the range $3.5<K_{0}<4.0$, and no stars brighter than $K_{0}=3.5$. In the range $4.0 \leq K_{0} \leq 6.0$, the GC and PGC LFs are based on 43 and 33 stars respectively. If the PGC LF were the same as the GC LF, we would expect $\sim 162 \pm 13$ stars in the range $4.0 \leq K_{0} \leq 6.0$ inside $\operatorname{Reg} 1$ and $\operatorname{Reg} 2$, which is inconsistent with the actual number observed at the $9 \sigma$ level. The individual half mag bins of the PGC LF are also deficient relative to the GC LF for all $K_{0}<6.0$.

The BW LF brighter than $K_{0}=7.0$ from Tiede et al. (1995) is based on a very small number of stars. Further, even these few bright stars with $K_{0}<7.0$ could be foreground stars (Tiede et al. 1995). We estimate the number of foreground stars expected in each half mag bin for the grism survey of $M$ giants used by FW87 for two different models of the disk LF. The FW87 field covers an area of $468 \mathrm{arcmin}^{2}$. The disk is assumed to have a radial scale length of $3 \mathrm{kpc}$ in both the models (Kent et al. 1991). The local density of different $M$ giants given by Garwood \& Jones (1987) is scaled appropriately to derive the density of these stars in any volume element along the line of sight to BW. In the first model, (GJ model) the disk scale height of all the 9 different spectral classes of $M$ giants is taken to be 300 pc (Garwood \& Jones 1987). In the second model, we assume a disk scale height of $165 \mathrm{pc}$ for all the spectral classes (Kent et al. 1991). The expected foreground contamination in the FW87 field for observation cones extending to distances of $4 \mathrm{kpc}$ and $6 \mathrm{kpc}$ are given in Table 4. This table also contains the number of $M$ giants that are actually observed by FW87 in each half mag interval. For $K_{0}<7.0$, the expected number of foreground $M$ giants toward the BW in both models is comparable to the actual number of stars that are observed in the FW87 field. On the other hand, we find that the the contamination of the PGC stars by foreground stars is negligible $(<0.15$ per half mag bin). To understand this result, note that the total observed flux in the PGC regions corresponds to $K \sim 13.4 \mathrm{mag} \operatorname{arcsec}^{-2}$, corresponding to a dereddened surface brightness of $K \sim 10.7 \mathrm{mag} \operatorname{arcsec}^{-2}$. This is about 70 times brighter than BW with $K \sim 15.3 \mathrm{mag} \operatorname{arcsec}^{-2}$. Thus, although the absolute surface density of foreground disk stars is higher in the PGC than BW, the ratio of disk to bulge stars 
is much smaller. Even if all the 6 stars brighter than $K_{0}=7.0$ detected in the BW grism surveys are taken to be bulge stars, the PGC LF still contains a significant excess over the BW LF when all the bins in the range $5.5<K<7.0$ are combined. The PGC LF is therefore different from both the LF of the inner $2^{\prime}$ of the GC and the BW LF. It lies intermediate between the LFs of the GC and the BW for all $K_{0}<7.0$.

\section{Discussion}

The stellar population of the GC has an excess of bright stars compared to the the older population of BW (Haller 1992). The central pc of the Galaxy contains helium

rich, luminous, blue, emission-line stars and Wolf-Rayet stars with estimated zero-age main sequence masses of up to $\sim 100 M_{\odot}$ (Allen, Hyland \& Hillier 1990; Krabbe et al. 1991; Blum, Sellgren, \& DePoy 1995a; Blum, DePoy, \& Sellgren 1995b; Libonate et al. 1995; Krabbe et al. 1995). A plausible scenario is a burst of star formation in the GC $\sim 10$ Myr ago (Krabbe et al. 1995). Krabbe et al. (1995) also conclude that the intermediate mass asymptotic giant branch stars were formed in another burst of star formation $\sim 100$ Myr ago.

In the PGC, we do not find stars that are as luminous as the brightest stars in the GC. Nevertheless, there is a significant excess of stars with $K_{0} \leq 7$ over the older population of the BW. This could imply the existence of a population of stars that is significantly younger than the old bulge population. The best way to confirm this hypothesis would be to take spectra of the bright stars in the PGC. To this end, we present in Table 5 a list of all stars with $K_{0}<5$ in the PGC region.

\section{Conclusion}

We have constructed the $\mathrm{K}$ band $\mathrm{LF}$ of the perigalactocentric region outside the inner $2^{\prime}$ of the GC, by individually dereddening every star using its observed $(H-K)$ colors. This LF is complete for $K_{0}<8$. Unlike the GC LF, this LF does not have any bright end $\left(K_{0}<4.0\right)$ component. There is also a deficiency in the PGC LF compared to the GC LF for all $K_{0} \leq 6$. However, the PGC LF has a significant excess over the $\mathrm{BW}$ LF for $K_{0} \leq 7$. We conclude that the PGC LF is intermediate between the GC and the BW LFs. 
We thank Bob Blum, Jay Frogel, Kris Sellgren and David Weinberg for helpful comments and suggestions. We also thank R. Bertram for his help with the observations. Work by A.G was supported in part by NSF grant AST-94-20746. OSIRIS was constructed with support from the NSF grants AST90-16112 and AST92-18449. 


\section{REFERENCES}

Allen, D. A., Hyland, A. R., \& Hillier, D. J., 1990, MNRAS, 244, 706

Blanco, V. M. 1986, AJ, 91, 290

Blanco, V. M., McCarthy, M. F., \& Blanco, B. M. 1984, AJ, 89, 636

Blum, R. D., Sellgren, K., \& DePoy, D.Ł. 1996, ApJ, submitted

Blum, R. D., Sellgren, K., \& DePoy, D.Ł. 1995a, ApJL, 440, L17

Blum, R. D., DePoy, D.Ł., \& Sellgren, K. 1995b, ApJ, 441, 603

DePoy, D. L., Atwood, B., Byard, P., Frogel, J. A., \& O'Brien, T., 1993, in SPIE 1946, "Infrared Detectors and Instrumentation," p.667

DePoy, D. L., Terndrup, D. M., Frogel, J. A., Atwood, B., \& Blum, R., 1993, AJ, 105,2121

Frogel, J. A., 1988, ARA\&A, 26, 51

Frogel, J. A., \& Whitford, A. E. 1987, ApJ, 320, 199

Garwood, R., \& Jones, T. J. 1987, PASP, 99, 453

Haller, J. 1992, Ph.D Thesis, University of Arizona,Tucson

Kent, S. M., Dame, T. M., \& Fazio, G. 1991, ApJ, 378, 131

Krabbe, A., Genzel, R., Drapatz, S., \& Rotaciuc, V., 1991, ApJL, 382, L19

Krabbe, A., et al. 1995, ApJ, 447, L95

Libonate, S., Pipher, J. L., Forrest, W. J., \& Ashby, M. L. N. 1995, ApJ, 439, 202

Mathis, J. S. 1990, ARA\&A, 28, 37

Stetson, P. B., 1987, PASP, 99, 191

Tiede, G., Frogel, J. A., \& Terndrup, D. M. 1995, AJ, 110, 2788 
Table 1: LFs and the number of stars actually observed in each half magnitude bin for the 2 sets of regions of PGC, the inner $2^{\prime}$ of GC and BW.

\begin{tabular}{ccccccccc}
\hline \hline$K_{0}$ & \multicolumn{4}{c}{ PGC } & \multicolumn{2}{c}{ GC } & \multicolumn{2}{c}{ BW $^{\dagger}$} \\
\cline { 2 - 5 } & \multicolumn{2}{c}{ REG1 } & \multicolumn{2}{c}{ REG2 } & & & \\
\cline { 2 - 5 } & LF & N & LF & N & LF & N & LF & N \\
\hline 1.75 & 0 & 0 & 0 & 0 & 0.46 & 1 & 0 & 0 \\
2.25 & 0 & 0 & 0 & 0 & 0.00 & 0 & 0 & 0 \\
2.75 & 0 & 0 & 0 & 0 & 0.91 & 2 & 0 & 0 \\
3.25 & 0 & 0 & 0 & 0 & 0.46 & 1 & 0 & 0 \\
3.75 & 0 & 0 & 0 & 0 & 1.83 & 4 & 0 & 0 \\
4.25 & 0.43 & 1 & 0 & 0 & 0.92 & 2 & 0 & 0 \\
4.75 & 0.43 & 1 & 0.54 & 4 & 3.66 & 8 & 0 & 0 \\
5.25 & 0.86 & 2 & 0.41 & 3 & 5.03 & 11 & 0 & 0 \\
5.75 & 3.47 & 8 & 1.90 & 14 & 10.05 & 22 & 2.56 & 1 \\
6.25 & 11.60 & 26 & $7.24^{*}$ & 53 & 10.51 & 23 & 0.92 & 1 \\
6.75 & 20.25 & 46 & 20.96 & 179 & 34.29 & 75 & 7.01 & 4 \\
7.25 & 31.80 & 71 & 38.63 & 328 & 53.03 & 116 & 51.22 & 14 \\
7.75 & $42.36^{*}$ & 94 & 58.50 & 495 & 64.10 & 139 & 52.12 & 15 \\
8.25 & 107.83 & 210 & 76.48 & 645 & 84.74 & 187 & 49.92 & 10 \\
8.75 & 161.09 & 274 & 91.67 & 772 & 97.83 & 214 & 104.77 & 14 \\
9.25 & 124.74 & 209 & 87.21 & 800 & 128.92 & 282 & 198.20 & 340 \\
9.75 & 133.63 & 215 & 99.84 & 916 & 146.29 & 320 & 287.50 & 505 \\
10.25 & 127.93 & 212 & 84.58 & 776 & 122.06 & 267 & 431.24 & 663 \\
10.75 & 156.64 & 196 & 80.88 & 742 & 65.37 & 143 & 574.98 & 925 \\
11.25 & 125.91 & 152 & 72.48 & 665 & 30.63 & 67 & 718.74 & 1309 \\
11.75 & 90.31 & 112 & 59.85 & 549 & 13.25 & 29 & 1006.23 & 1786 \\
\hline
\end{tabular}

$\dagger$ For $5 \leq K_{0} \leq 9$, the photometry is from FW87. For $9 \leq K_{0} \leq 12$, the photometry is from DePoy et al. (1993).

* Stars in this bin and brighter were examined individually. For fainter mag, we applied statistical corrections. For $9 \leq K_{0} \leq 12$, the photometry is from DePoy et al. (1993). 
Table 2: Reg 1 patches.

\begin{tabular}{cccc}
\hline \hline Patch id & $\begin{array}{c}\Delta \alpha \\
(")\end{array}$ & $\begin{array}{c}\Delta \delta \\
(")\end{array}$ & $\begin{array}{c}\text { Area } \\
\left(\operatorname{arcmin}^{2}\right)\end{array}$ \\
\hline 1 & 397.5 & -422.5 & 1.98 \\
2 & 249.9 & -277.1 & 1.85 \\
3 & 134.8 & -221.6 & 2.51 \\
4 & 140.6 & -89.8 & 2.54 \\
5 & -139.0 & 205.3 & 2.25 \\
6 & -248.3 & 324.9 & 1.71 \\
\hline
\end{tabular}


Table 3: Reg2 patches.

\begin{tabular}{cccc}
\hline \hline Patch id & $\begin{array}{c}\Delta \alpha \\
(")\end{array}$ & $\begin{array}{c}\Delta \delta \\
(")\end{array}$ & $\begin{array}{c}\text { Area } \\
\left(\operatorname{arcmin}^{2}\right)\end{array}$ \\
\hline 1 & 129.1 & -437.6 & 1.83 \\
2 & -116.6 & -407.9 & 2.40 \\
3 & -261.6 & -409.6 & 2.30 \\
4 & -125.5 & -126.5 & 1.95 \\
5 & -198.1 & -248.7 & 1.80 \\
6 & -333.9 & -244.3 & 2.29 \\
7 & -224.5 & -29.9 & 1.84 \\
8 & -381.1 & -122.8 & 2.37 \\
9 & -357.5 & 95.6 & 2.71 \\
10 & -374.9 & 273.2 & 4.93 \\
11 & -435.2 & 123.9 & 2.69 \\
12 & 460.7 & -314.5 & 2.38 \\
13 & 351.5 & -183.6 & 2.49 \\
14 & 424.6 & -2.9 & 3.26 \\
15 & 458.0 & -146.5 & 1.98 \\
16 & 220.0 & 57.3 & 1.10 \\
17 & 306.3 & 225.3 & 2.64 \\
18 & 470.7 & 128.0 & 2.05 \\
19 & 340.3 & 293.9 & 2.61 \\
20 & 184.2 & 302.4 & 2.73 \\
21 & -43.6 & 378.2 & 2.69 \\
22 & 474.3 & 228.1 & 2.21 \\
\hline
\end{tabular}


Table 4: Expected number of foreground $M$ giants towards BW in the grism survey of FW87 for 2 different models of the star distribution, assuming distances to the bulge of 4 and $6 \mathrm{kpc}$. GJ model (Garwood and Jones 1987) and Kent model (Kent et al. 1991).

\begin{tabular}{cccccc}
\hline \hline MAGNITUDE & \multicolumn{2}{c}{ GJ MODEL } & \multicolumn{2}{c}{ KENT MODEL } & NUMBER OBSERVED \\
\cline { 2 - 5 } & $4 \mathrm{kpc}$ & $6 \mathrm{kpc}$ & $4 \mathrm{kpc}$ & $6 \mathrm{kpc}$ & \\
\hline 4.25 & 0.10 & 0.10 & 0.07 & 0.07 & 0 \\
4.75 & 0.20 & 0.22 & 0.13 & 0.14 & 0 \\
5.25 & 0.34 & 0.47 & 0.22 & 0.27 & 0 \\
5.75 & 0.58 & 0.77 & 0.35 & 0.43 & 1 \\
6.25 & 0.95 & 1.47 & 0.55 & 0.76 & 1 \\
6.75 & 1.05 & 2.60 & 0.61 & 1.20 & 4 \\
7.25 & 1.58 & 4.20 & 0.88 & 1.80 & 14 \\
7.75 & 1.70 & 3.70 & 0.92 & 1.70 & 15 \\
8.25 & 1.44 & 6.30 & 0.77 & 2.60 & 10 \\
8.75 & 1.57 & 6.50 & 0.79 & 2.50 & 14 \\
\hline
\end{tabular}


Table 5: Photometry of stars in PGC with $K_{0} \leq 5$.

\begin{tabular}{ccccccc}
\hline \hline ID & $\begin{array}{c}\Delta \alpha \\
(")\end{array}$ & $\begin{array}{c}\Delta \delta \\
(")\end{array}$ & $K$ & $(H-K)$ & $K_{0}$ & $(H-K)_{0}$ \\
\hline 1 & -80.4 & -489.3 & 9.58 & 4.53 & 3.92 & 0.75 \\
2 & 133.4 & -243.9 & 9.41 & 3.92 & 4.56 & 0.68 \\
3 & 255.0 & 379.9 & 10.17 & 4.36 & 4.64 & 0.67 \\
4 & 98.3 & -284.7 & 10.93 & 4.79 & 4.74 & 0.66 \\
5 & 286.3 & -439.9 & 7.73 & 2.60 & 4.81 & 0.65 \\
6 & -228.8 & -106.4 & 10.83 & 4.62 & 4.87 & 0.65 \\
7 & 118.0 & 390.8 & 7.86 & 2.63 & 4.88 & 0.65 \\
8 & 406.3 & -127.2 & 7.75 & 2.55 & 4.89 & 0.65 \\
9 & 134.9 & -281.1 & 10.82 & 4.59 & 4.90 & 0.64 \\
10 & -226.9 & 57.8 & 8.12 & 2.79 & 4.90 & 0.64 \\
11 & -446.0 & 169.9 & 8.85 & 3.26 & 4.91 & 0.64 \\
12 & -507.5 & -16.3 & 8.77 & 3.21 & 4.93 & 0.64 \\
13 & -284.5 & 113.6 & 8.61 & 3.09 & 4.94 & 0.64 \\
14 & 137.5 & 209.2 & 8.00 & 2.67 & 4.95 & 0.64 \\
15 & -157.7 & 136.4 & 7.55 & 2.36 & 4.96 & 0.64 \\
16 & 438.1 & 290.8 & 8.98 & 3.30 & 4.98 & 0.64 \\
\hline
\end{tabular}




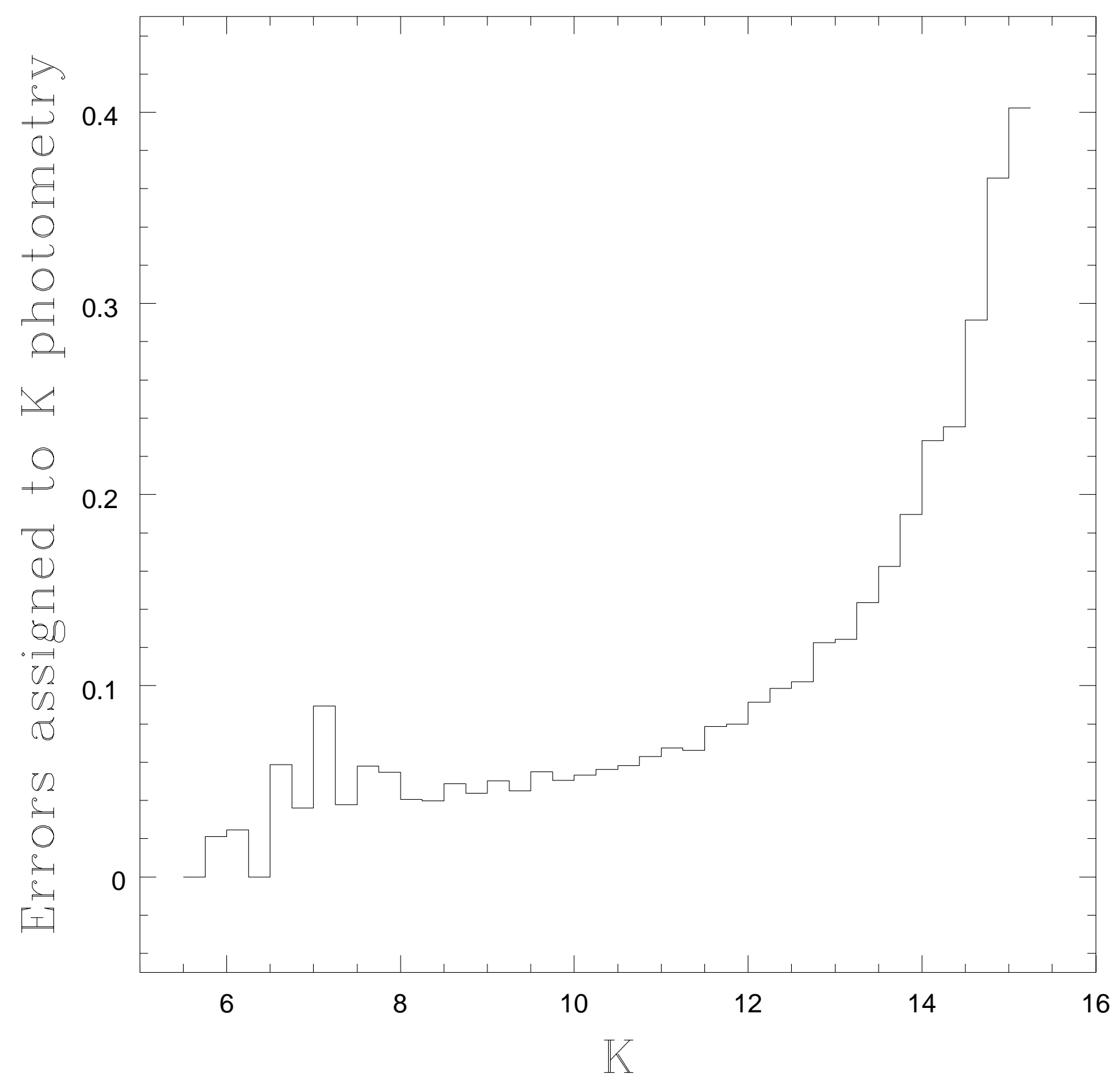

Figure 3: Errors in the K Photometry. 


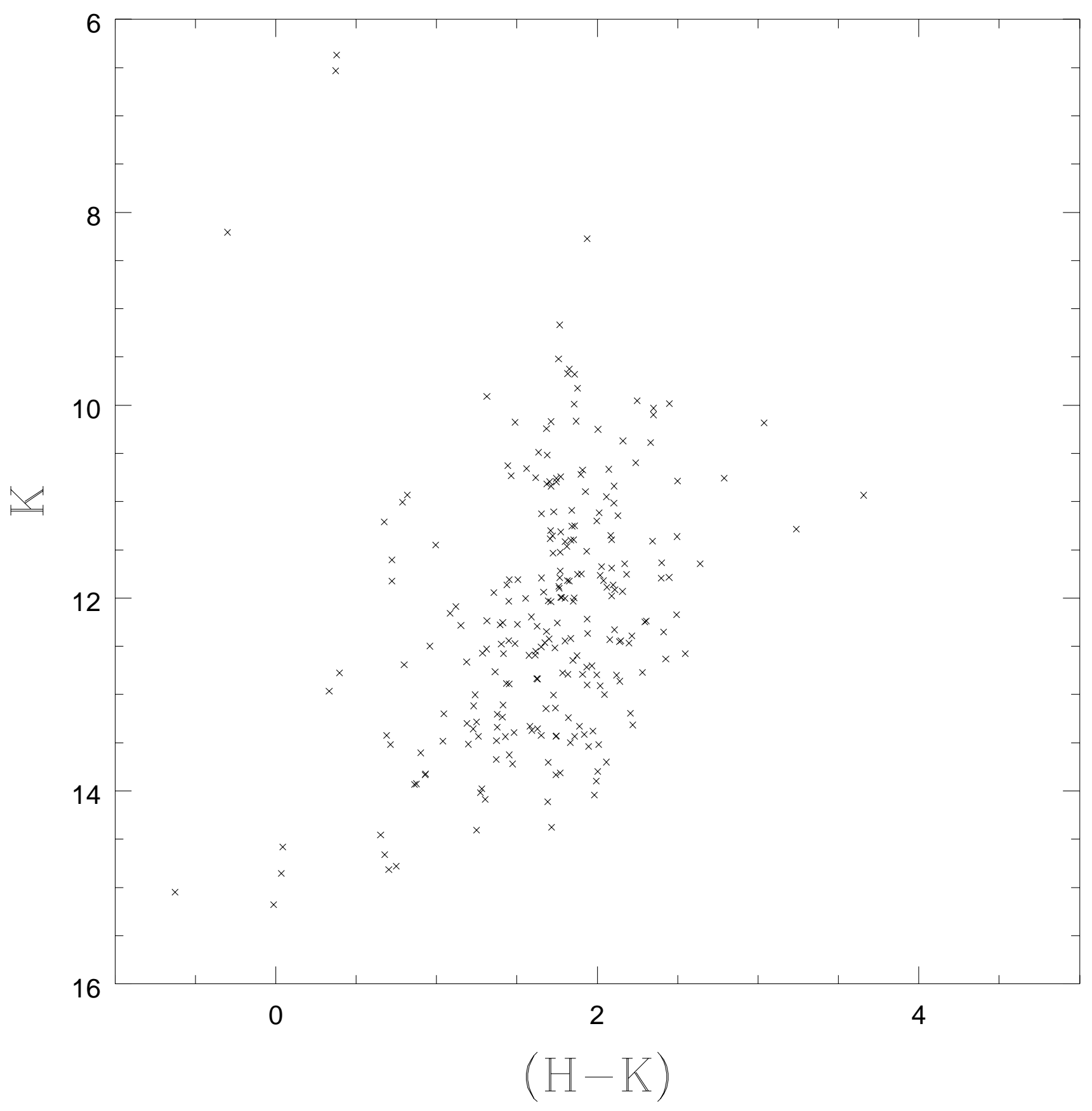

Figure 4: Color-mag diagram of patch 1 of Reg1. 


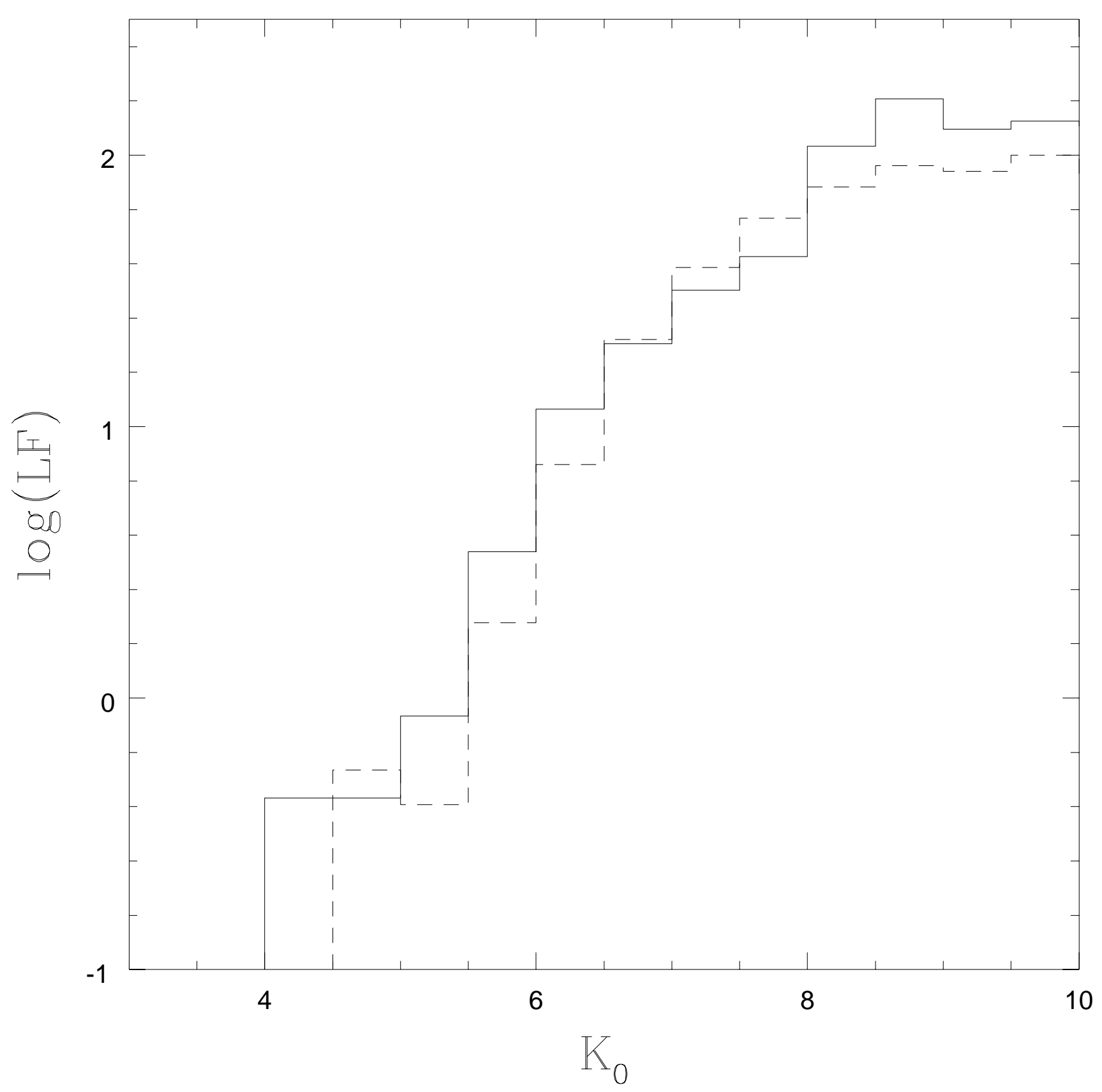

Figure 5: Comparison of the LFs from two different regions of PGC. Reg1 (solid line) and Reg2 (dashed line). The two LFs are identical within Poisson errors. 


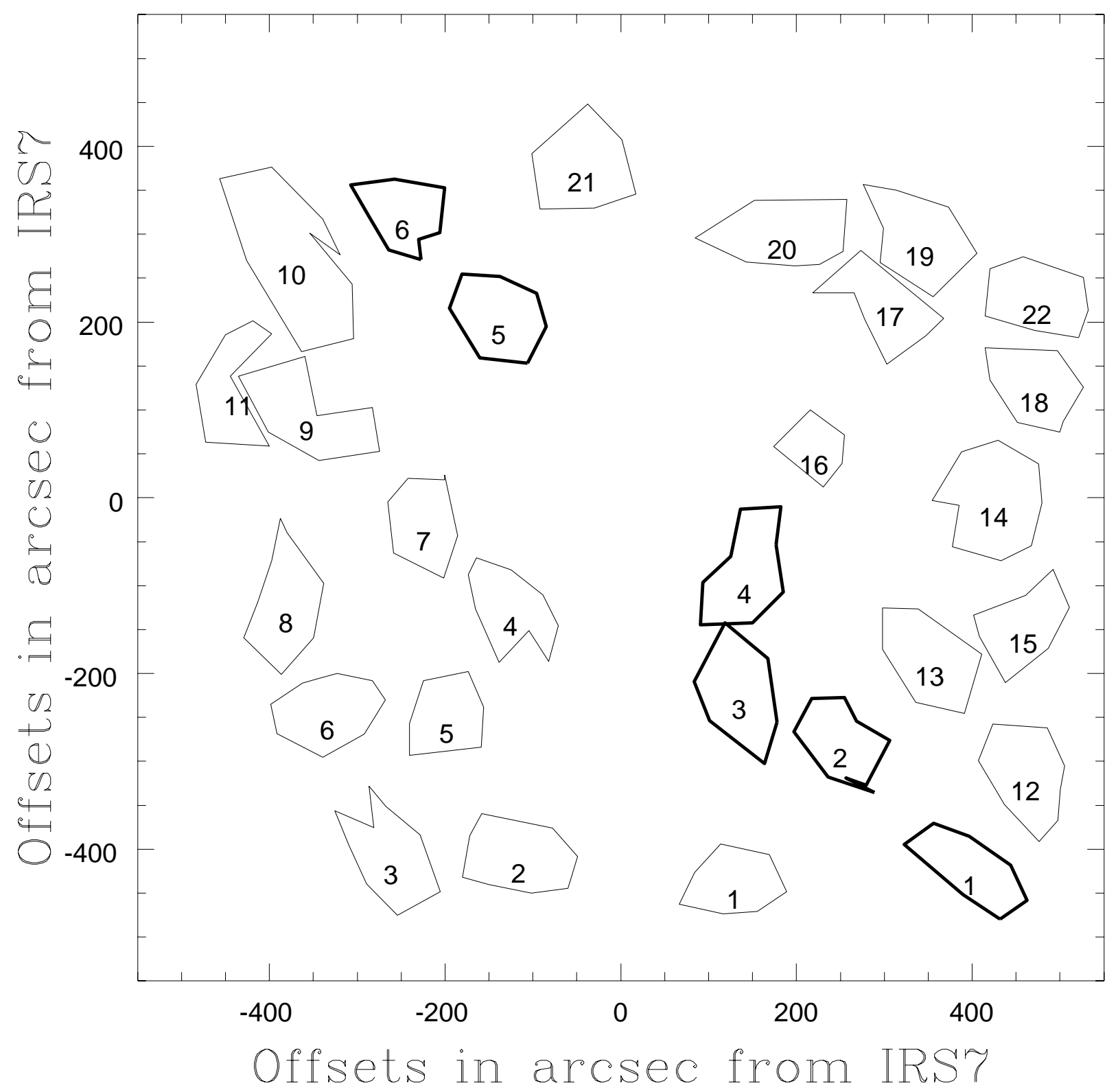

Figure 6: The 6 regions comprising Reg1(thick lines) and the 22 regions comprising Reg2 (ordinary lines). North is up and east is left. Reg1 patches lie in the disk like feature in the $\mathrm{K}$ mosaic, while the Reg2 regions are outside it. 


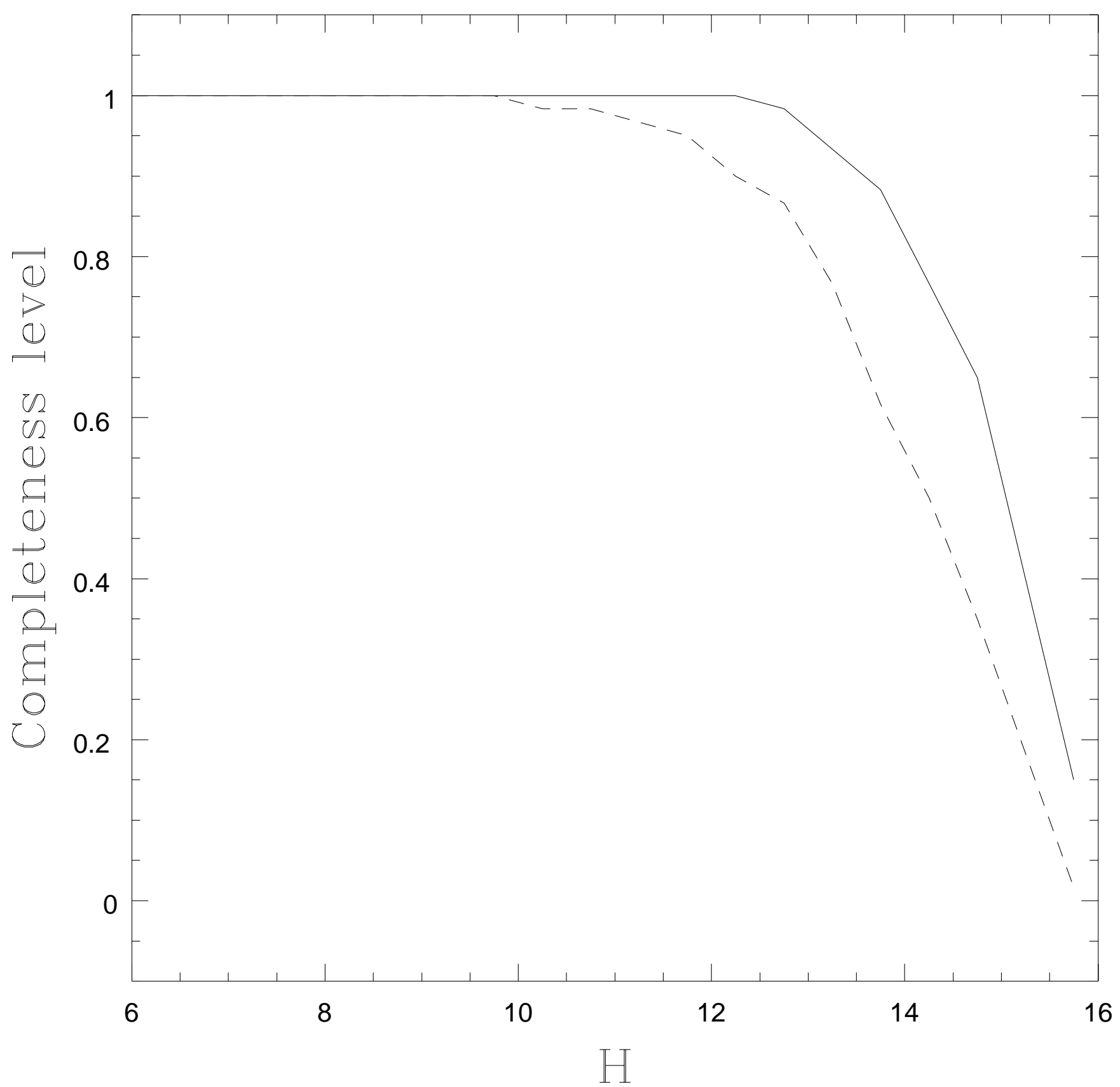

Figure 7: Completeness level as a function of $H$ for the total star list (solid line) and the DAOPHOT star list (dashed line). 


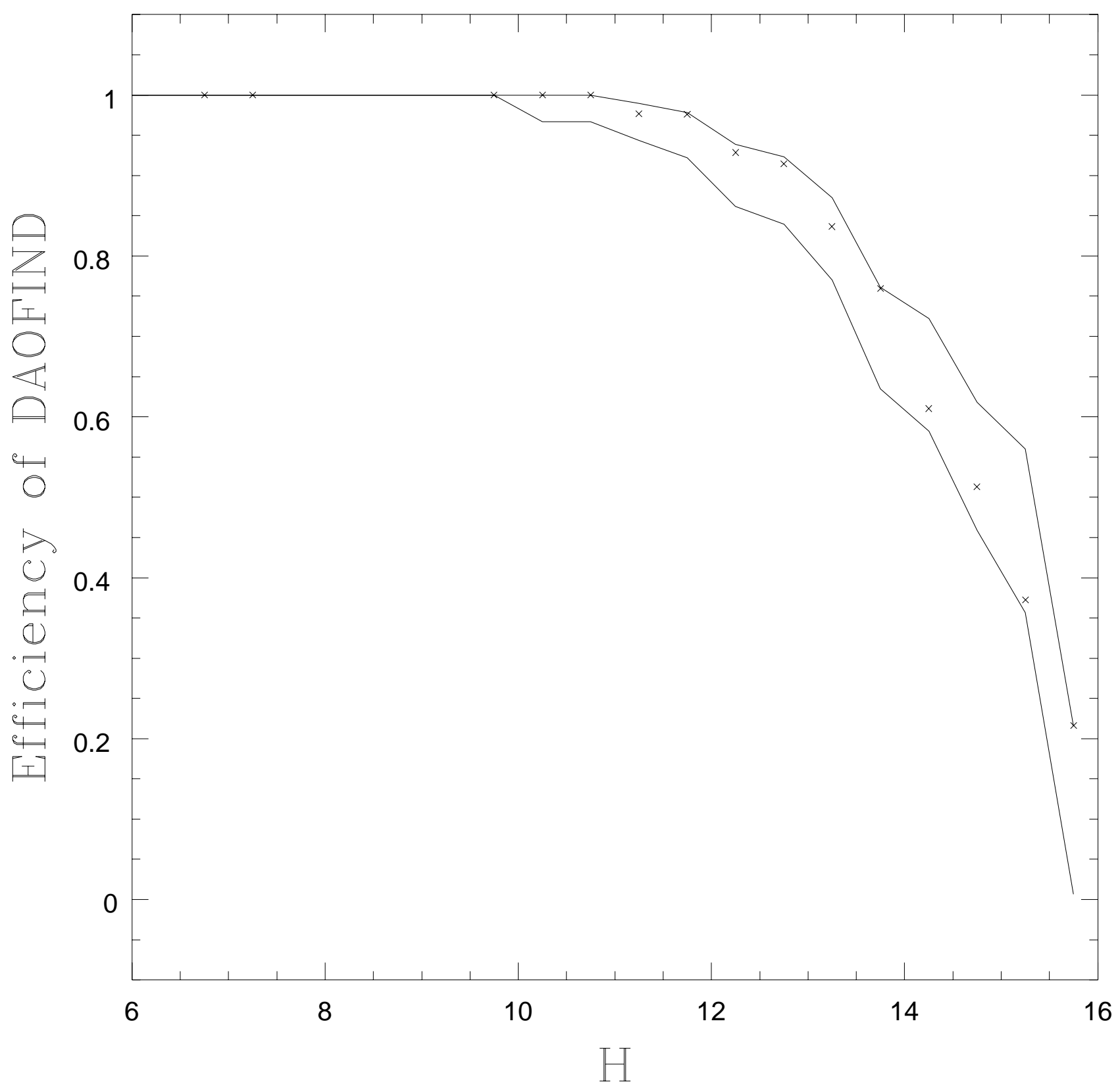

Figure 8: The two solid curves enclose the $1 \sigma$ bound of the DAOPHOT detection fraction determined from the artificial star tests. The points are the fraction for the sample star list. 


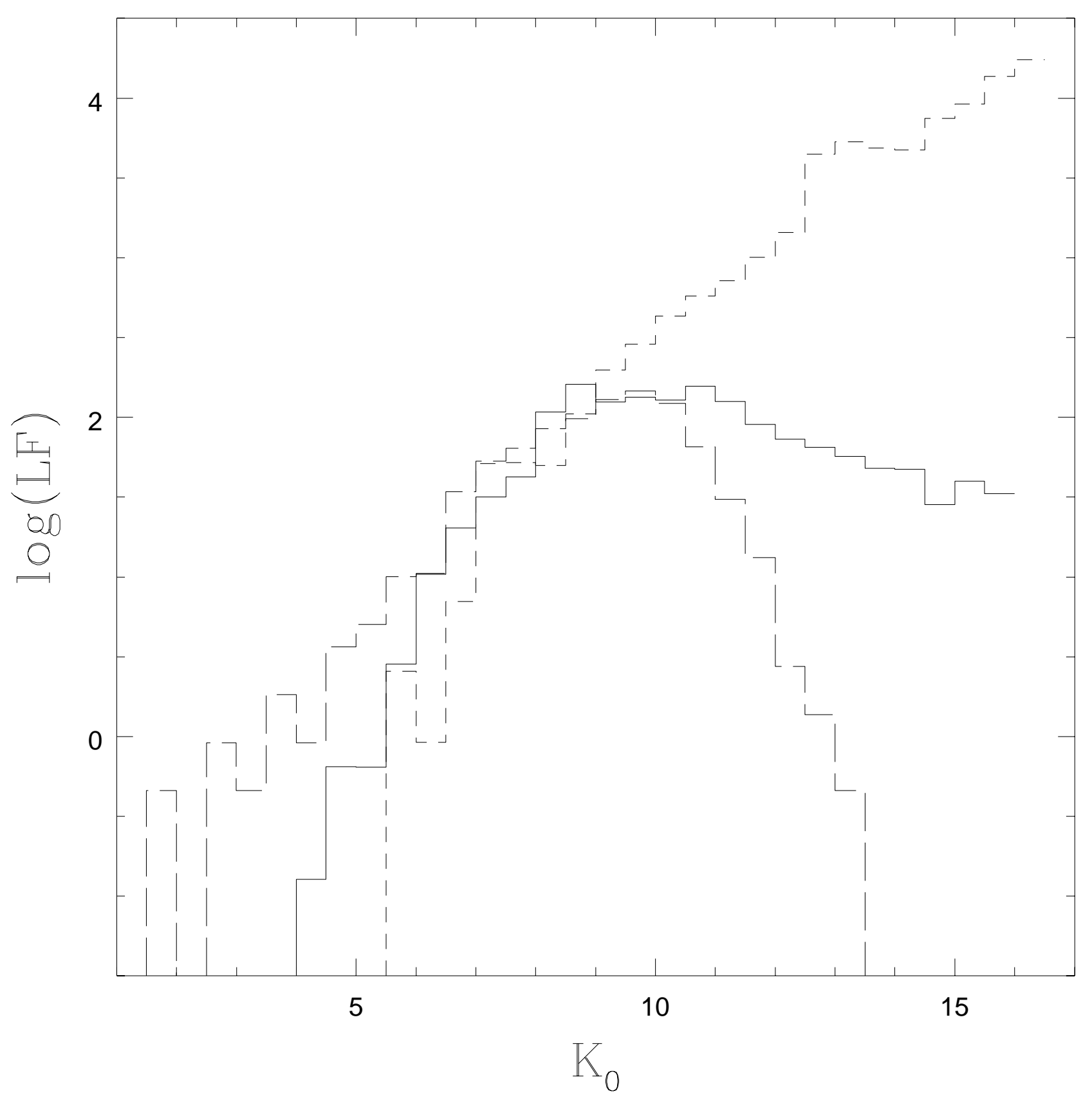

Figure 9: LFs of PGC (solid line), GC (long dashed line) and BW (short dashed line). For $K_{0}<6.5$, the PGC LF is constructed from both Reg1 and Reg2. 